\title{
No Work for a Theory of Universals
}

\author{
M. Eddon \\ C. J. G. Meacham
}

\begin{abstract}
Several variants of Lewis's Best System Account of Lawhood have been proposed that avoid its commitment to perfectly natural properties. There has been little discussion of the relative merits of these proposals, and little discussion of how one might extend this strategy to provide natural property-free variants of Lewis's other accounts, such as his accounts of duplication, intrinsicality, causation, counterfactuals, and reference. We undertake these projects in this paper. We begin by providing a framework for classifying and assessing the variants of the Best System Account. We then evaluate these proposals, and identify the most promising candidates. We go on to develop a proposal for systematically modifying Lewis's other accounts so that they, too, avoid commitment to perfectly natural properties. We conclude by briefly considering a different route one might take to developing natural property-free versions of Lewis's other accounts, drawing on recent work by Williams (this volume).
\end{abstract}

\section{$1 \quad$ Introduction}

In "New Work for a Theory of Universals," David Lewis proposes a version of the Best System Account of Lawhood: a law is a sentence entailed by the best system; the best system is the set of true sentences that best balances simplicity and informativeness, and whose predicates refer to only the perfectly natural properties and relations. ${ }^{1}$

Many philosophers find the Best System Account of Lawhood appealing, at least in broad outline. ${ }^{2}$ But Lewis's version of the Best System Account requires a

\footnotetext{
${ }^{1}$ Lewis restricts the laws to regularities entailed by the best system. But many of his successors have dropped this requirement, thus allowing laws constraining initial conditions (for example, see Loewer (2001), Hoefer (2007), and Winsberg (2008)).

${ }^{2}$ Loewer (2007) writes: "I am very much attracted to [the Best System Account] because of the way it incorporates the criteria physicists use for counting generalizations and equations as expressing laws and also because it, unlike many of its rivals, doesn't posit metaphysically primitive laws, primitive causal powers, propensities, governing relations, or other metaphysically heavy-duty and suspect entities." (2007, 313) Callender and Cohen (2009) echo the sentiment that the Best System Account is appealing in part because it is not "metaphysically freighted": "It is grounded in nothing more than the properties, individuals, and events in the world, and formal (deductive) relations defined over statements about these entities, that are already recognized by our best scientific descriptions of the way the world is. The modesty of [the Best System Account's] extrascientific apparatus has made the view seem attractive to thinkers who are inclined to defer to the best scientific descriptions of the world - both to Humeans (and others who forswear necessary
} 
primitive distinction between properties that are perfectly natural and those that are not. And those who find a Best System Account attractive because it does not posit mysterious or inscrutable primitive causal powers, governing relations, and the like, tend to be unhappy with this appeal to perfectly natural properties. ${ }^{3}$ For, one might say, the distinction Lewis relies on is no less mysterious or inscrutable.

Is there a way to develop a Best System Account of Lawhood that preserves the features that these philosophers find appealing, and does not invoke a primitive distinction among properties? Although a number of people have discussed this question, the literature on this topic is somewhat disjointed. Different parties focus on different issues, and set up the dialectic in different ways.

This paper has three goals. The first is to provide a framework for the debate. We lay out the space of available positions on this question, pinpoint the crucial junctures, and show how participants in the debate can be seen as taking different stances at these junctures.

The second goal is to evaluate the prospects of these different positions, and to identify the most promising candidates. After surveying the options, we argue that there are reasons to prefer a version of the Best System Account of Lawhood suggested by Loewer (2007) and Hoefer (2007) over others.

The third goal is to broaden the horizons of the original project. Perfectly natural properties appear not only in Lewis's analysis of lawhood, but also in his accounts of objective resemblance, duplication, intrinsicality, causation, counterfactuals, certain supervenience claims, and reference. Is there a way to modify these accounts, so that they too can do without perfect naturalness? We suggest one way to do this using the version of the Best System Account discussed above.

The paper proceeds as follows. In section (2), we present the basic form of the Best System Account, and sketch some ways this account may be developed. Using this framework, we lay out the landscape of proposals that people have offered. In section (3), we discuss some of the worries facing the different proposals, and select the options that strike us as the most promising. In section (4), we consider the question of whether one can modify Lewis's other accounts as well, so that they too do not appeal to perfectly natural properties. We develop one proposal, and consider some objections. Finally, in section (5), we briefly

connections) and more generally to those who prefer fewer metaphysical posits to more." (2009, 2 3)

${ }^{3}$ For example, see Taylor (1993), Roberts (1999), Halpin (2003), Hoefer (2007), Loewer (2007), and Callender and Cohen (2009). 
consider a different route one might take, drawing on work by Williams (this volume).

\section{Best System Accounts of Laws}

Let's begin by sketching a basic template for a Best System account of lawhood. To keep things simple, we will generally restrict our attention to non-probabilistic laws.

Consider the sets of true sentences. Some of these sets are more informative than others - they tell us more about what the world is like. Some of these sets are simpler than others - they contain fewer or shorter sentences. These two features tend to compete with one another. Adding more sentences to a set may increase its informativeness, but decreases simplicity; removing sentences may decrease informativeness, but increases simplicity. Now consider the set of true sentences that does best, on balance, at satisfying the desiderata of simplicity and informativeness. Call this set the Best System. Anything entailed by the Best System is a law of nature. ${ }^{4}$

This template is silent on two key questions. The first question has to do with the language in which sentences of candidate systems are formulated. ${ }^{5}$ Consider Lewis's example of a language containing the predicate F, where F applies to all and only the things at the actual world. ${ }^{6}$ The set containing only the sentence $\forall x \mathrm{~F} x$ is maximally simple (it contains only one short sentence) and maximally informative (it entails every truth about the actual world). If it is permissible to formulate systems in a language containing $\mathrm{F}$, then we get the absurd result that $\{\forall x \mathrm{~F} x\}$ is the best system, and every truth is a law. In order to rule out a system like this, we need to place some constraints on the languages employed by candidate systems. So what language should be used to formulate candidate systems? Call this the Language Question.

The second question has to do with our evaluation of how well candidate systems satisfy the relevant desiderata. There are a number of ways one might

\footnotetext{
${ }^{4}$ We follow Lewis ([1983] 1999, 41-42) in remaining neutral between understanding laws as propositions or interpreted sentences.

${ }^{5}$ Languages may take many forms. Following Lewis, we restrict our attention to languages that take the form of something like second-order predicate logic. Moreover, these languages are interpreted languages. (If they weren't interpreted, one would not be able to evaluate the informativeness of sentences in the language.)

${ }^{6}$ Lewis (1983)
} 
assess the simplicity or informativeness of a set of sentences. One metric of simplicity might take some logical operators to add more to a sentence's complexity than others - maybe material implication adds more complexity than negation. One metric of informativeness might take certain sentences to be more informative than others, even though neither sentence entails the other - maybe the sentence describing the future history of the universe is more informative than the sentence describing what I ate for breakfast. Thus, which system is best, and $a$ fortiriori what the laws are, may vary depending on the metrics of simplicity, informativeness, balance, and whatever else we deem relevant. So which metrics should we use to evaluate candidate systems? Call this the Metrics Question.

Different answers to these questions yield different Best System accounts. In the following sections, we briefly describe the answers to these questions that have been offered in the literature. We then sketch the landscape of the literature, laying out the space of positions taken by various authors.

\subsection{Answers to the Language Question}

The Language Question: What language should we use to formulate candidate systems?

\section{Language-Objectivity: an objective, “mind-independent" language}

This is Lewis's preferred answer to the Language Question; on his view, the language of candidate systems is the one containing only those predicates that refer to the perfectly natural properties. This language is objective or "mindindependent" because which properties are perfectly natural is an objective matter, having nothing to do with us.

\section{Language-Indexicality: the language of the speaker}

According to Language-Indexicality, when one asserts that $P$ is a law, one's claim is true iff, when the candidate systems are formulated in one's language, the best system entails $P$. (Alternatively, one might hold that the appropriate language of candidate systems is the language of the society to which the person belongs, or perhaps the language of that society's scientists.)

\section{Language-Rigidity: our language}

According to this answer, we take the language of candidate systems to be our language. (Or, alternatively, the language of our society, or our society's scientists, or some idealized version of our society's scientists.) Furthermore, when 
considering candidate systems of other possible worlds, we formulate these systems in our actual language - not the language we would have had at the world under consideration. In other words, we "rigidify" our language.

\section{Language-Relativity: any language - and Best Systemhood is language- relative}

A fourth option is to allow any language to be used to formulate candidate systems, and make "best-systemhood" - and hence lawhood - relative to a language. So, for any language $L$, candidate systems-in- $L$ vie for Best System-in$L$. Consider two languages, $L_{1}$ and $L_{2}$. Suppose that $P$ is entailed by the Best System-in- $L_{1}$ at world $w$, and $Q$ is entailed by the Best System-in- $L_{2}$ at $w$. Then $P$ is a law-in- $L_{1}$ at $w$, and $Q$ is a law-in- $L_{2}$ at $w$, but neither $P$ nor $Q$ is a law simpliciter.

\section{Language-Salience: all languages - and the Best System must be salient}

A fifth option is to push the burden of choosing a language elsewhere. Here is one way to do that. Consider all candidate systems, formulated in all possible languages. In choosing among candidate systems, we consider not only simplicity and informativeness, but also whether a system is unified, useful, and explanatory - for short, salient. On the Language-Salience answer, the language of the Best System is the language of the system that best balances simplicity, informativeness, and salience. Adding this desideratum allows us to rule out systems such as $\{\forall x \mathrm{~F} x\}$; for while $\{\forall x \mathrm{~F} x\}$ scores highly on simplicity and informativeness, it scores very low on salience. ${ }^{7,8}$

\subsection{Answers to the Metrics Question}

The Metrics Question: What metrics of simplicity, informativeness, balance, and whatever else we deem relevant, should we use when evaluating candidate systems?

\section{Metrics-Objectivity: the objective, "mind-independent" metrics}

The Metrics-Objectivity answer says that there is an objectively correct way to measure the extent to which a system satisfies the specified criteria (e.g., simplicity, informativen ess, balance, etc.)

\footnotetext{
${ }^{7}$ See section (2.3) for more on how to understand the salience desideratum.

${ }^{8}$ See Loewer (2007, 324-325).
} 


\section{Metrics-Indexicality: the metrics of the speaker}

According to Metrics-Indexicality, when one asserts that $P$ is a law, one's claim is true iff, when candidate systems are assessed using the metrics of the speaker, the system judged best entails $P$. (Alternatively, one might hold that candidate systems should be evaluated using the metrics of the society to which the person belongs, or perhaps the metrics of that society's scientists.)

\section{Metrics-Rigidity: our metrics}

Another option is to take the metrics used to evaluate the extent to which candidate systems satisfy the specified criteria to be our metrics. (Or, alternatively, the metrics of our society, or our society's scientists, or some idealized version of our society's scientists.) As with the Language-Rigidity answer, "our metrics" should be understood rigidly - when considering candidate systems of other possible worlds, we assess these systems using our actual metrics, not those we would have had at the world under consideration.

\section{Metrics-Relativity: any metrics - and Best Systemhood is metrics-relative}

On the Metrics-Relativity answer, we evaluate candidate systems using any metrics, and make "best-systemhood" relative to the metrics employed. So, for any set of metrics $M$, there is a best system-evaluated-by- $M$. Consider two sets of metrics, $M_{1}$ and $M_{2}$. Suppose that $P$ is entailed by the Best System-evaluated-by$M_{1}$ at $w$, and $Q$ is entailed by the Best System-evaluated-by- $M_{2}$ at $w . P$ is a lawevaluated-by- $M_{1}$, and $Q$ is a law-evaluated-by- $M_{2}$, but neither is a law simpliciter.

\subsection{The Landscape}

Below is a chart summarizing the different positions that authors have taken in recent literature. Because some authors defend positions that are compatible with multiple answers, their names appear more than once. 


\section{Metrics Question}

\begin{tabular}{|c|c|c|c|c|c|}
\hline & \multicolumn{1}{|c|}{ Objectivity } & Indexicality & Rigidity & Relativity \\
\cline { 2 - 6 } & Objectivity & Lewis (1994) & Lewis (1994) & $\begin{array}{c}\text { Lewis (1980) } \\
\text { Lewis (1994) }\end{array}$ & Lewis (1994) \\
\cline { 2 - 6 } & Indexicality & & $\begin{array}{c}\text { Roberts (1999) } \\
\text { Halpin (2003) }\end{array}$ & & \\
\begin{tabular}{l} 
Question \\
\cline { 2 - 6 }
\end{tabular} & Rigidity & Loewer (2007) & & $\begin{array}{c}\text { Hoefer (2007) } \\
\text { Loewer (2007) }\end{array}$ & \\
\cline { 2 - 6 } & Relativity & Taylor (1993) & & $\begin{array}{c}\text { Taylor (1993), } \\
\text { Halpin (2003), } \\
\text { Callender \& } \\
\text { Cohen (2009) }\end{array}$ \\
\cline { 2 - 6 } & Salience & Loewer (2007) & & $\begin{array}{c}\text { Hoefer (2007) } \\
\text { Loewer (2007) }\end{array}$ & \\
\hline
\end{tabular}

Barry Taylor (1993) and Craig Callender and Jonathan Cohen (2009) defend the Language-Relativity answer to the Language Question, though they differ on points of detail. Their answers to the Metrics Question are less straightforward. Callender and Cohen do not explicitly endorse any answer to the Metrics Question, though Metrics-Relativity seems to best fit the spirit of their proposal. ${ }^{9}$ And Taylor seems open to both Metrics-Relativity and MetricsObjectivity. ${ }^{10}$ In general, however, all of them take the laws to be relative, and all of them take there to be no deep sense in which the laws relative to one relatum are privileged over the laws relative to some other relatum.

John F. Halpin (2003) offers what he calls a "contextual" or "perspectival" account of lawhood. On this account, lawhood is relativized to perspectives, and the appropriate perspective is selected by context. ${ }^{11}$ He can be read as endorsing either the Relativity answers to both questions or the Indexicality answers to both

\footnotetext{
${ }^{9}$ Callender and Cohen's discussion suggests that they take Language-Relativity to provide an answer to the Metrics Question as well as the Language Question. For instance, they write: "[We assess] the immanently strongest, simplest, and best balanced axiomatizations relative to a specific choice of basic kinds $K$ (to a specific choice of basic predicates $P_{K}$ ). Given such an assessment relative to a choice of basic kinds $K$ (predicates $P_{K}$ ), we can say that a true generalization is a law relative to $K\left(P_{K}\right)$ just in case it appears in all the immanently Best Systems relative to the basic kinds $K$ (basic predicates $\left.P_{K}\right)$." $(2009,21)$

${ }^{10}$ See Taylor $(1993,97)$.

${ }^{11}$ See Halpin (2003, 151 and 156).
} 
questions. $^{12}$

John Roberts (1999) suggests that the best way to develop a Best System analysis of lawhood invokes Metrics-Indexicality. ${ }^{13}$ Although he does not directly address the Language Question, it seems to be in the spirit of his account to endorse Language-Indexicality as well. Thus Roberts's discussion suggests the Indexicality answers to both questions.

Barry Loewer (2007) suggests two answers to the Language Question. One is Language-Salience: we consider candidate systems in all possible languages, and add a desideratum which takes in to account other scientific virtues besides simplicity and informativeness - such as usefulness and explanatory value. ${ }^{14}$ The other is Language-Rigidity: the language of candidate systems is some suitably idealized version of the actual language of our scientific community. ${ }^{15}$ As for the Metrics Question, Loewer believes that our standards of simplicity, informativeness, and salience are deeply rooted in our scientific tradition - what we find explanatory, useful, and so on. But he also believes that it's plausible that these notions may be at least partially objective. ${ }^{16}$ This suggests some combination of Metrics-Rigidity and Metrics-Objectivity: in cases where the objective metrics do not select a unique candidate system as best, we appeal to those of the actual scientific community.

Although Carl Hoefer (2007) focuses on a Lewisian account of chance, rather than a Lewisian account of laws, his position fits neatly into this framework. Hoefer defends a position similar to Loewer's, adopting Metrics-Rigidity and some combination of Language-Salience and Language-Rigidity. But while Hoefer's

\footnotetext{
${ }^{12}$ For passages that suggest the Indexicality answers, see pages 142,149 , and 151 . For passages that suggest the Relativity answers, see pages 156 and 163-164.

${ }^{13}$ However, Roberts does not commit himself to any version of the Best System account. In his (2009), Roberts provides a detailed defense a different, though still Humean, account of laws. 14 "From the perspective of the aims of science the obvious trouble with ' $(\mathrm{x}) \mathrm{Fx}$ ' is not that ' $\mathrm{Fx}$ ' doesn't refer to a perfectly natural property but that ' $(\mathrm{x}) \mathrm{Fx}$ ' is not a credible scientific theory. It is completely lacking in explanatory value. ... The information in a theory needs to be extractable in a way that connects with the problems and matters that are of scientific interest." (Loewer 2007, 324-325)

15 "Here is a proposal. Let SL be a present language of science, say scientific English... A candidate for a final theory is evaluated with respect to, among the other virtues, the extent to which it is informative and explanatory about truths of scientific interest as formulated in SL or any language SL+ that may succeed SL in the rational development of the sciences." (Loewer 2007, 325)

16 "[P] art of what makes a truth a law is to an extent anthropomorphic since it involves human criteria of simplicity and informativeness and... explanatoriness and 'scientific interest.' But these may well be relatively objective notions." (Loewer 2007, 325) "It may turn out that the [Final Theory] entails that any intelligent creature that has a concept that plays a role like the concept of law plays for us will evaluate simplicity and informativeness in ways very similar to us." (Loewer 2007, 327)
} 
treatment of salience is similar to Loewer's in spirit, it differs in letter. Instead of treating salience as an additional desideratum, Hoefer incorporates salience directly in to the simplicity and informativeness requirements. Thus he suggests we understand the (salient)-simplicity desideratum as selecting for something like user-friendliness, taking comprehen sibility and ease of use for agents like us into consideration. And he suggests that we understand the (salient)-informativeness desideratum as selecting for something like helpfulness, taking utility and relevance to agents like us in to consideration. ${ }^{17}$

Finally, David Lewis endorses Language-Objectivity - appealing to perfectly natural properties to determine the language of candidate systems (Lewis (1983) and (1994)). Lewis's position on the Metrics Question is less clear. In earlier work, Lewis (1980) adopts Metrics-Rigidity: we employ our metrics when assessing candidate systems, and we hold these fixed when assessing systems at other possible worlds. ${ }^{18}$ But he later revises his position. ${ }^{19}$ His remarks in Lewis (1994) suggest two different responses to the Metrics Question. One option is Metrics-Objectivity. Lewis writes: "I suppose our standards of simplicity and strength and balance are only partly a matter of psychology ... maybe some of the exchange rates between aspects of simplicity, etc. are a psychological matter, but not just anything goes. If nature is kind, the best system will be robustly best - so far ahead of its rivals that it will come out first under any standards of simplicity and strength and balance." ([1994] 1999, 232-233) And if nature is unkind, and no system is clearly best, then "the theorems of the barely-best system would not very well deserve the name of laws." (233) This amounts to the Objectivity answer to the Metrics Question, since only the objective metrics play a role in determining the laws. If nature is kind, then the objective metrics select the unique best system; if nature is unkind, and the objective metrics do not select a

\footnotetext{
${ }^{17}$ See Hoefer (2007, 571-572).

18 "The standards of simplicity, of strength, and of balance between them are to be those that guide us in assessing the credibility of rival hypotheses as to what the laws are. In a way, that makes lawhood depend on us - a feature of the approach that I do not at all welcome! But at least it does not follow that lawhood depends on us in the most straightforward way: namely, that if our standards were suitably different, then the laws would be different. For we can take our actual standards as fixed, and apply them in asking what the laws would be in various counterfactual situations, including counterfactual situations in which people have different standards - or in which there are no people at all. Likewise, it fortunately does not follow that the laws are different at other times and places where there live people with other standards." (Lewis [1980] 1986, 123)

19 "I used to think rigidification came to the rescue: in talking about what the laws would be if we changed our thinking, we use not our hypothetical new standards of simplicity and strength and balance, but rather our actual and present standards. But now I think that is a cosmetic remedy only. It doesn't make the problem go away, it only makes it harder to state." (Lewis [1994] 1999, 232)
} 
unique best system, then there are no laws.

The other option suggested by Lewis is a combination of MetricsObjectivity with some other answer to the Metrics Question. Lewis writes: "I can admit that if nature were unkind, and if disagreeing rival systems were running neck-and-neck, then lawhood might be a psychological matter, and that would be very peculiar." ([1994] 1999, 233) So, at a world where the objective metrics do not select one system as clearly best, then the metrics we use to evaluate candidate systems would be partly determined by objective evaluative facts, and partly determined by facts about our psychology. This option amounts to some combination of Metrics-Objectivity with one of the other answers. ${ }^{20}$

\section{$3 \quad$ Assessing the Options}

Proponents of Best System accounts of laws have offered a number of different answers to the Language and Metrics Questions. But how do these different answers rate against one another? In this section, we discuss the challenges facing each of these answers, and offer our assessment of the options.

\subsection{Language-Objectivity and Metrics-Objectivity}

Let's begin with the two Objectivity answers. According to Language-Objectivity, there is an objectively correct, "mind-independent" language in which candidate systems are formulated. According to Metrics-Objectivity, there is an objectively correct, "mind-independent" set of metrics that determines how candidate systems score with respect to their balance of simplicity and informativeness (and whatever else).

It seems likely that those who are skeptical of Lewis's distinction between natural and non-natural properties will be skeptical of both the Objectivity answers as well. For appealing to an objectively correct language or set of metrics is (arguably) no less mysterious than appealing to a primitive distinction between natural and non-natural properties. Since this paper is addressed to those disinclined to adopt natural properties, we will put both Language-Objectivity and Metrics-Objectivity aside.

\footnotetext{
${ }^{20}$ One might wonder why Metrics-Rigidity is included among the answers that entail that lawhood is a "psychological matter." For it might seem that the laws are dependent upon one's psychology iff the following counterfactual is true: had one's psychology been appropriately different, then the laws would have been different. If so, then the Metrics-Rigidity answer would not make the laws dependent on one's psychology. Lewis, however, rejects this construal of psychological dependence, and maintains that there is a sense in which Metrics-Rigidity makes the laws dependent on our psychology (see Lewis $(1994,232)$ ).
} 


\subsection{Language-Indexicality and Metrics-Indexicality}

The Indexicality answers take the term "law" to be an indexical, like "here" and "now." But whereas the referents of "here" and "now" are determined by the spatial and temporal location of the speaker, the referent of "law" is determined by the language and metrics of the speaker. ${ }^{21,22}$ For simplicity, our discussion focuses on Metrics-Indexicality, though what we say applies to both Indexicality answers. ${ }^{23}$

There are a several worries facing the Indexicality answers. One worry is that, if the term "law" is an indexical, then there is not enough room for meaningful disagreement about lawhood. ${ }^{24}$ Suppose Aristotle and Newton are having a conversation about what the laws of nature are. And suppose that Aristotle and Newton speak the same language, but have different metrics of simplicity, informativeness, and balance: the best system indexed to Aristotle's metrics yields Aristotelian Mechanics, whereas the best system indexed to Newton's metrics yields Newtonian Mechanics. It seems that Aristotle and Newton should be able to meaningfully disagree about what the laws are. But suppose Aristotle says, "The laws are Aristotelian Mechanics," and Newton replies by saying, "The laws are Newtonian Mechanics." Given the Indexicality answers, this is no more of a disagreement than Aristotle saying, "I am at the North Pole," and Newton saying, "I am in Massachusetts." For when Aristotle says, "The laws

${ }^{21}$ There are "narrow" and "broad" versions of the Indexicality answers. On the narrow understanding, the language or metrics are indexed to the speaker. On the broad understanding, the language or metrics are indexed to the speaker's scientific community. For ease of discussion, we assume the narrow understanding in the text, although nothing hangs on this.

Similarly, there are "actual" and "ideal" versions of the Indexicality answers. On the actual understanding, the language or metrics are indexed to the actual language or metrics of the party in question (either the speaker or the speaker's community). On the ideal understanding, the language or metrics are indexed to some idealized version of that party. In the section assessing the Rigidity answers, we argue against the idealized understanding of these answers, and the same arguments apply here as well. For ease of discussion, we assume the actual understanding in the text.

${ }^{22}$ A somewhat related view takes "laws" to pick out the sentences entailed by the best system indexed to the language and metrics of the individual assessing a given utterance, rather than indexed to those of the speaker. We discuss this view in footnote (28).

${ }^{23}$ Our discussion can be adapted to address Language-Indexicality in the following way. Let us say that Aristotle and Newton speak distinct but overlapping languages. The languages share terms such as "law," "language," etc. - whatever is needed so that they can intelligibly converse about laws, and make sense of sentences (1)-(5). But the languages differ with respect to their other predicates, in such a way that Aristotle's laws differ from Newton's. The worries raised in the text then apply to Language-Indexicality.

${ }^{24}$ Thanks here to Sinan Dogramaci. For an interesting reply to these kinds of worries, see Roberts (2009, section 3.3) (though Roberts is evaluating these worries with respect to a somewhat different account of laws). 
are Aristotelian Mechanics," the term "law" refers to those given by Aristotelian Mechanics, whereas when Newton says, "The laws are Newtonian Mechanics," the term "law" refers to those given by Newtonian Mechanics. Aristotle and Newton are not disagreeing; they are just talking past each other.

Another worry for taking "law" to be an indexical term concerns the truthvalues of counterfactuals involving embedded claims. Consider again Aristotle and Newton. If Aristotle says:

(1) If I were at the North Pole and I were to say, "It's cold here," then my assertion would be true,

then what he says is true. It does not matter whether Aristotle is actually in the Sahara Desert. The claim, "It's cold here," is evaluated with respect to the world at which he's at the North Pole; and at that world, it's cold at the North Pole.

Likewise, treating "law" as an indexical like "here," if Aristotle says:

(2) If I had Newton's metrics and I were to say, "The laws are Newtonian Mechanics," then my assertion would be true, then what he says is true. For the claim, "The laws are Newtonian Mechanics," is evaluated at the world where Aristotle has Newton's metrics; and at that world, his utterance is true. Intuitively, however, counterfactuals like (2) are false. But the Metrics-Indexicality answer entails that (2) is true.

One might try to avoid this result by maintaining that embedded claims in counterfactuals are evaluated using the speaker's actual metrics. But if one makes this move, then one cannot truly assert certain consequences of the Indexicality view. For instance, if Newton says "The laws are Newtonian Mechanics," then his assertion is true. But if Aristotle says:

(3) If Newton says "The laws are Newtonian Mechanics," then his assertion is true,

then his assertion is false. For on the present suggestion, we evaluate the embedded claim, "The laws are Newtonian Mechanics," using Aristotle's actual metrics. And Aristotle's metrics entail that the laws are not Newtonian Mechanics. So even though it is true that if Newton says "The laws are Newtonian Mechanics," then his assertion is true, this move entails that many of us who don't share Newton's metrics are unable to say that it's true. That seems wrong - surely those who don't share Newton's metrics can still truly assert (3). (Indeed, we've done so ourselves in the third sentence of this paragraph!)

The fact that the Indexicality answers deliver counterintuitive results for certain counterfactual claims reveals one way in which these answers appear to make the laws dependent on us in some important sense. But there is an even more straightforward way in which the Indexicality answers make the laws of 
nature us-dependent: if "law" is an indexical term, then the truth-values of sentences concerning the laws vary depending on who the speaker is. And that is a strange result. For whatever we say about what the laws are, it seems that they aren't the sorts of things that should vary from speaker to speaker.

\subsection{Language-Rigidity and Metrics-Rigidity}

According to the Language-Rigidity and Metrics-Rigidity answers, we use our language and metrics, understood rigidly, to determine the laws. ${ }^{25,26}$ These

${ }^{25}$ Do the Rigidity answers differ from a version of the Objectivity answers according to which the objectively correct language and metrics "happen" to line up with our own? It depends. If we allow ourselves a notion of "in virtue of" or "dependence," then we can articulate the difference between the two views in the following way: on the Rigidity answers, the right language and metrics to use depend on us; on the Objectivity answers, they do not (and the fact that the objectively correct language and metrics happen to line up with our own is just a coincidence). If we do not allow ourselves a notion of "in virtue of" or "dependence," then the two views seem to be equivalent. One can then understand our discussion as spelling out some worries for this particular version of the Objectivity answers (although the "arbitrariness" worry mentioned in footnote (32) may be more naturally construed as a "coincidence" worry).

${ }^{26}$ One could understand the phrase "our language and metrics" in either a non-egalitarian way (there's some particular community, ours, whose language and metrics are used to determine the laws) or an egalitarian way (every community uses its own language and metrics to determine the laws). The view we discuss in the text adopts the non-egalitarian reading, since that's the view discussed in the literature. But the egalitarian reading is interesting enough to be worth a few remarks. On the egalitarian version of the Rigidity answers, the members of a community assess nomic claims using the language and metrics of their community; so the same utterance may be true when assessed by a member of one community and false when assessed by a member of another. Thus on this egalitarian view the truth values of nomic claims are relative to the assessor's community assessing them. This is an instance of the kind of Relativism described by MacFarlane (2005), (2009), (2013a), and (2013b).

The egalitarian version of the Rigidity answers faces several worries. First, like the view discussed in the text, it seems likely that we will assess the nomic claims made by future scientists as false. (They won't be false when assessed by the future scientists, of course, but it is still counterintuitive that we should entertain these kinds of doubts about the nomic claims made by future scientists.) Second, since the same utterance can have different truth values when assessed by different people (see MacFarlane (2005)), this view requires one to accept a kind of relativism about truth that some may find unappealing.

Finally, as we note in the section assessing the Relativity answers, lawhood is tied to many other notions (causation, dispositions, reference, mental states, etc.), and if laws are assessordependent, then these other notions are assessor-dependent as well. As a result, adopting assessordependence about lawhood implies that many more claims are assessor-dependent than advocates of relative truth generally believe. For recently proposed assessor-dependent views tend to be circumscribed to, say, epistemic modals, knowledge attributions, or claims about taste (see MacFarlane (2009), (2013a), and (2013b)). If we adopt assessor-dependence, about lawhood, then not only are nomic claims assessor-dependent, but so are causal claims, counterfactual claims, dispositional claims, claims about reference (given a causal theory of reference), claims about psychological states (given a functionalist theory of mind), and the like. 
answers can be understood in either a narrow way - as picking out the language and metrics of a particular individual - or a broad way - as picking out the language and metrics of an individual's scientific community. Since proponents of Rigidity answers generally adopt a broad understanding, that is what we assume in what follows.

Begin with the Language-Rigidity answer, according to which candidate systems are formulated in the language of our scientific community. This answer has some unpalatable consequences. The language of the scientific community today differs substantially from that of the scientific community one thousand years ago. And it seems likely that in one thousand years from now, the scientific community will be speaking of properties presently unknown to us, and using unfamiliar terminology from fields of mathematics yet to be developed. And so it seems reasonable to expect that the language of future scientists will have little in common with our current language, and that the laws posited by future scientists will be formulated in a language quite different from the one we currently use. If that's the case, and candidate systems are to be formulated in the language of our current scientific community, then future scientists are likely to get the laws wrong. But we generally think that future scientists are in a better position to discern the laws of nature, not a worse position. ${ }^{27}$

To avoid this result, an advocate of Language-Rigidity might say that the language of a candidate system is not our language, but rather an ideal version of our language: the language that an idealized version of our scientific community, in possession of all the non-nomic facts, would use to formulate their final scientific theory. ${ }^{28}$ But this appeal to the ideal language and, by extension, the idealized scientific community, raises other worries.

One worry arises from the counterfactual nature of this approach. In order for $\mathrm{L}$ to be the ideal language, something like the following counterfactual must be true:

(4) If our scientific community were idealized, and were to know all the nonnomic facts, then they would use L to formulate their final scientific theory.

\footnotetext{
${ }^{27}$ See also Carroll (1990, 201-202).

${ }^{28}$ Loewer (2007) suggests something like this move. He characterizes the "final theory" (FT) in this way: "FT maximally satisfies all the requirements that the tradition and practice of fundamental physics puts on a fundamental theory of the world. FT is true, simple, highly informative, comprehensive; FT reconciles relativity and quantum theory, explains statistical mechanical probabilities, and explains special science regularities, and so on. It does all this better than any alternative theory whether the alternative has ever been or ever will be thought up by anyone. There is no true theory that better than FT balances all these virtues." $(2007,322)$
} 
But the truth value of such counterfactuals is generally taken to depend on what the laws are. ${ }^{29}$ So this appeal to the ideal language threatens to make the account circular.

An other worry arises from the characterization of the ideal language as the one used by the idealized scientific community. The language an idealized scientific community will use to formulate their final theory will be one that makes their final theory attractive - simple, unified, perspicuous, and so on. If it's legitimate for our account of laws to appeal to the language this idealized scientific community would use to construct their final theory, then it should be legitimate for our account to appeal to the final theory this idealized scientific community would construct. But then we might as well appeal to the final theory directly: let the laws be whatever the final theory says they are.

But surely this is not a legitimate move. Appealing to an idealized scientific community just shifts the burden from describing how to get the laws from the non-nomic facts to describing how an idealized scientific community would construct their final theory from the non-nomic facts. The task in both cases is essentially the same - characterizing a function that takes the non-nomic facts as input, and spits out an attractive final theory as output. And re-describing the task in terms of idealized scientific communities doesn't make it any easier.

Now let's turn to Metrics-Rigidity. Like Language-Rigidity, this answer has an "actual" understanding (rigidify our current, actual metrics) and an "ideal" version (rigidify those of an ideal scientific community). The idealized version of Metrics-Rigidity runs into the same troubles as the idealized version of LanguageRigidity. The non-idealized version of Metrics-Rigidity, on the other hand, seems to be on firmer ground than the non-idealized version of the Language-Rigidity answer. For while it's plausible that the language of the future scientific community will bear little resemblance to our current language, it's less plausible that the metrics of the future scientific community will bear little resemblance to our metrics of simplicity, informativeness, balance, etc. And thus the worry that arose for the non-idealized version of Language-Rigidity - that future scientists are likely to get the laws wrong because their language will differ from ours - does not seem as pressing for the non-idealized version of Metrics-Rigidity.

A more general worry, applicable to both the Language-Rigidity and Metrics-Rigidity answers, is that they threaten to make the laws of nature depend

\footnotetext{
${ }^{29}$ This is true on virtually every account of counterfactuals on offer; see Lewis (1979) and Maudlin (2007) for two examples at the opposite ends of the spectrum. (One also finds this close connection between laws and counterfactuals in Lange (2009), though he inverts the direction of dependence.)
} 
on us in some sense. For in appealing to our language and metrics, the Rigidity answers imply that the laws depend on the language and metrics of our scientific community. But intuitively, the laws of nature should not depend on what the language and metrics of any particular scientific community are like. ${ }^{30}$

This worry is hard to make precise, however. ${ }^{31}$ One natural way to assess whether the laws depend on us - our language and metrics - is to apply a counterfactual test: the laws depend on us iff had our language and metrics been appropriately different, the laws would have been different as well. But on this construal of "us-dependence," the Rigidity answers do not imply that the laws depend on what we are like. ${ }^{32}$ So to press this worry, one needs some other way of understanding the manner in which the Rigidity answers are "us-dependent." 33 Without that, it is unclear that this is a mark against the Rigidity answers.

In any case, the Rigidity answers seem better off than the Indexicality answers with respect to certain kinds of counterfactual claims. For unlike the

\footnotetext{
${ }^{30}$ Another way of developing this worry is to maintain that appealing to our language and metrics makes the laws seem "arbitrary" - why, out of all possible scientific communities, is ours the one whose language and metrics determine what the laws are? See Roberts (1999, S503).

31 "The worst problem about the best-system analysis is that when we ask where the standards of simplicity and informativeness come from, the answer may seem to be that they come from us.... I used to think rigidification came to the rescue... But now I think that is a cosmetic remedy only. It doesn't make the problem go away, it only makes it harder to state." (Lewis [1994] 1999, 232)

${ }^{32}$ For example, given that our community's language is $\mathrm{M}$ and the corresponding laws are $\mathrm{LAWS}_{\mathrm{M}}$, the Rigidity answers entail that counterfactuals like the following are false:
}

(5) If our community's language were $\mathrm{N}$, then the laws would not be $\mathrm{LAWS}_{\mathrm{M}}$. And this seems to be the right result. But one might wonder whether they run into trouble with respect to indicative conditionals. For example, the Rigidity answers allow for indicative conditionals like the following to be true:

(6) If our community's language is $\mathrm{M}$, then the laws are $\mathrm{LAWS}_{\mathrm{M}}$, but if our community's language is $\mathrm{N}$, then the laws are not $\mathrm{LAWS}_{\mathrm{M}}$.

We do not think these indicative conditionals reveal a disturbing kind of "us-dependence," since indicative conditionals are largely epistemic (see Kratzer (1986)). For example, suppose you know that the Objectivity answers to the Language and Metrics Questions are correct, and you know that the objectively correct language and metrics happen to line up exactly with those of your community. However, you don't know exactly what your community's language and metrics are. Then you could truly assert something like (2). But it doesn't follow that the Objectivity answers make the laws "us-dependent"! (Thanks here to Daniel Nolan and Louis deRosset.)

${ }^{33}$ Here is one attempt. Suppose, as in footnote (27), we allow ourselves some notion of "in virtue of" or "dependence." Then one can spell out the worry in this way: the Rigidity answers entail that the laws of nature obtain in virtue of us having the language and metrics that we do, and that makes the laws unacceptably dependent upon us.

But the notion of "in virtue of" is controversial, and not everyone will accept it: Lewis, for instance, claims that we do not have a "clear enough understanding of 'solely in virtue of."' (2001, 384) If the foremost proponent of perfect naturalness rejected this notion as too mysterious, then it's plausible that those who reject naturalness as too mysterious will as well. And if so, they will not be moved by this way of putting the worry. 
Indexicality answers, they deliver the desired results when it comes to counterfactuals like (2).

\subsection{Language-Relativity and Metrics-Relativity}

Next let's consider the two Relativity answers: if one adopts either of the Relativity answers, then the notion of lawhood is relativized - to a language (given Language-Relativity), a set of metrics (given Metrics-Relativity), or both.

It is commonly noted that our concept of lawhood is deeply related to modal notions, causal notions, and many other philosophical concepts. ${ }^{34}$ If lawhood is a relation between a system and a language, or a system and a set of metrics, then this relativity will seep into these other notions as well. For example, although philosophers disagree on the details, it is generally thought that a vase can not be fragile without certain counterfactuals about it being true, that one cannot perceive the breaking of the vase without bearing some kind of causal relationship to it, and that one cannot explain the breaking of the vase without describing some of the causal or nomic facts that led to its breaking. If lawhood is relative to a language or set of metrics, then it seems that the notions of fragility, perception, and explanation must all be language or metrics relative as well.

One worry about the Relativity answers is that the relativity of lawhood will spread to many of our other notions. And it seems prima facie implausible that nomic claims, causal claims, counterfactual claims, dispositional claims (such as claims about fragility), claims about what one is referring to (given a causal theory of reference), claims about psychological states (given a functionalist theory of mind), and so on, are language or metrics relative.

A more troubling worry is that this relativization leads to normative conflicts. (The following examples focus on Language-Relativity, though the same worries may be raised for Metrics-Relativity.) Suppose that agents ought to align their credences with what they believe the chances to be, in accordance with Lewis's Principal Principle (1980). If the laws determine the chances, and the laws are language-relative, then the chances are language-relative as well. But this yields conflicting prescriptions about what we should believe. For instance, if I know that the laws relative to language $L_{1}$ assign a chance of 0.5 to a coin toss landing heads, and I know that the laws relative to language $L_{2}$ assign a chance of 0.6 to the same coin toss landing heads, then it seems that I am required to have a

\footnotetext{
${ }^{34}$ See, for instance, Carroll (1994, 1-12).
} 
credence of both 0.5 and 0.6 in the coin toss landing heads. ${ }^{35,36}$

Another example: causal decision theorists maintain that, roughly, a prudentially rational agent will perform the act that she expects to causally bring about the most utility. But if the causal facts are language-relative, then how much utility an act causally brings about is also language-relative. And thus it seems that causal decision theory yields conflicting prescriptions regarding what we ought (prudentially) to do. For example, consider a scenario where the causal facts relative to language $L_{1}$ are such that smoking will cause cancer, while the causal facts relative to language $L_{2}$ are such that smoking is only statistically correlated with cancer. Assuming that one knows all of this, and that one finds smoking enjoyable, it seems that causal decision theory will prescribe both smoking and not smoking.

Similar worries arise for the normative prescriptions of moral theories like utilitarianism, which typically evaluate the net change in utility brought about by an act by evaluating the causal influence of that act. And legal prescriptions run into trouble as well. Suppose a company dumps toxins near a town's water supply, and the town experiences a spike in cancer rates. The company is legally obligated to pay compensation to the town iff the toxins it dumped nearby are the cause of this increase in cancer rates. Consider two languages, $L_{1}$ and $L_{2}$, such that the toxins cause-in- $L_{1}$ cancer but do not cause-in- $L_{2}$ cancer. Then the law yields conflicting prescriptions about whether the company owes the town any compensation.

Now, one might attempt to avoid these normative conflicts by relativizing norms as well. But this would require a wholesale revision of how we think about norms. And one might be wary of upending our conception of normativity in order to hold on to a particular account of laws.

\subsection{Language-Salience}

Finally, let's consider Language-Salience. According to this answer, candidate systems may be formulated in any language whatsoever. In order to rule out

\footnotetext{
${ }^{35}$ This is an overly simplified description of the case; as described, there are still moves the proponent of relativization can make. For a more detailed and rigorous description of cases in which such conflicts arise, see Meacham (2014).

${ }^{36}$ Yet another worry here is that, given a functionalist causal role account of belief, the beliefs one has are themselves relativized to a language. For instance, the causal facts given language $L_{1}$ might be such that one has a credence of 0.5 in $\mathrm{P}$, while the causal facts given language $L_{2}$ might be such that one does not have a credence of $0.5 \mathrm{in}$ P. Thus even if the chance of a coin landing heads is 0.5 relative to both $L_{1}$ and $L_{2}$, an agent who satisfies the Principal Principle relative to $L_{1}$ will fail to do so relative to $L_{2}$, since $L_{1}$ and $L_{2}$ assign her different credences.
} 
"trivial" candidate systems (for instance, a system consisting just of $\{\forall x \mathrm{~F} x\}$, which is maximally informative and simple), we add a third desideratum: salience. A salient system is useful, unified, and explanatory. Because $\{\forall x \mathrm{~F} x\}$ scores very low on salience, it cannot be the Best System.

One might worry that the Language-Salience answer makes the laws of nature depend on us. For it is natural to think that assessments of salience whether a system is useful, explanatory, etc. - implicitly rely on certain facts about we are like - what we find useful, explanatory, etc. If so, appealing to salience risks making the laws depend on particular features of our psychologies.

Note that this worry applies equally well to simplicity and informativeness. It may seem that assessments of simplicity and informativeness implicitly rely on certain facts about what we are like - what we find simple and informative. And if so, appealing to simplicity and informativeness risks making the laws depend on particular features of our psychologies.

But whether appealing to any of these desiderata makes the laws depend on us in this way hinges on how we answer the Metrics Question. Given MetricsObjectivity, there are objective metrics of simplicity, informativen ess, and salience that are independent of us - and so the laws are independent of us. Given Metrics-Indexicality, on the other hand, the metrics of simplicity, informativeness, and salience are those of the speaker - and so the laws depend on the speaker. So there is nothing about the Language-Salience answer, in and of itself, that threatens to make the laws depend on us. Language-Salience just inherits the "us-dependence" of whatever answer to the Metrics Question it is paired with.

A more pressing worry for Language-Salience is that the notion of salience is somewhat nebulous and underspecified. While one might raise the same complaint for simplicity and informativeness, it's plausible that salience is worse off in this regard. (Though see Hoefer $(2007,571-572)$ for reasons to think that simplicity and informativeness are not nearly as clear as generally supposed. ${ }^{37}$ ) For

\footnotetext{
37 “' $[\mathrm{C}]$ loser inspection of Lewis's theory destroys the initial impression of tidiness. Simplicity and strength are meant to be timeless, objective notions unrelated to our species or our scientific history. But one suspects that if BSA advocates aim to have their account mesh with scientific practice, these notions will have to be rather pragmatically defined. Moreover, simplicity and strength are simply not clearly characterized by Lewis or his followers. We do not know whether initial conditions, giving the state of the world at a time (or a sub-region), should count as one proposition or as infinitely many (nor how to weigh the reduction of simplicity, whatever answer we give); we do not know whether deterministic laws are automatically as strong as can be, or whether instead some added chance-laws may increase strength at an acceptable price; if the latter, we do not know how to weigh the increase in strength so purchased." (Hoefer 2007, 571)
} 
one might maintain that we have more of an intuitive grasp on the notions of simplicity and informativeness than we have on the notion of salience. And while proponents of Language-Salience have gestured towards ways of understanding the notion, it is reasonable to expect a more concrete characterization.

\subsection{Taking Stock}

All of the alternatives to the Objectivity answers to the Language and Metrics Questions face challenges of one kind or another. Given that we've put the Objectivity answers aside, which of the remaining answers are the most promising?

Begin with the Language Question. Language-Indexicality has trouble making room for disagreement, delivers counterintuitive results regarding counterfactuals, and, because the truth-values of nomic claims vary from speaker to speaker, makes the laws "us-dependent" in some sense. Language-Relativity leads to normative conflicts, and entails that the many notions intertwined with lawhood must be relativized as well. For these reasons, both Language-Indexicality and Language-Relativity strike us as unappealing.

Language-Rigidity has drawbacks as well, though they differ depending on the version we're considering. The idealized version of Language-Rigidity faces serious worries stemming from its appeal to an idealized scientific community. The actual version strikes us as more viable, but it too has uncomfortable results. First, there is the worry that future scientists are likely to be mistaken about what the laws are. Second, there is the worry that the laws depend on what we're like in some sense. Still, the latter worry seems less pressing for Language-Rigidity than Language-Indexicality - for at least Language-Rigidity delivers the intuitively correct results regarding certain kinds of counterfactual claims.

Language-Salience seems to us to be the least problematic answer to the Language Question. The main worry facing this answer is that we do not have a fleshed out characterization of the notion of "salience" - and different characterizations of the notion may make this answer more or less plausible. Nonetheless, the task of providing a characterization seems feasible.

Next let's turn to the Metrics Question. Metrics-Indexicality and MetricsRelativity have the same demerits as their Language counterparts, and so are unappealing for the same reasons. And, as before, the actual version of MetricsRigidity strikes us as more viable than the idealized version. But unlike LanguageRigidity, the worry that future scientists will be wrong about what the laws are seems less likely to arise for Metrics-Rigidity. All in all, this answer seems to be the most promising option. 
The pair of answers that strike us as the most attractive - LanguageSalience and Metrics-Rigidity - yield a Best System account similar to the one advocated by Loewer (2007) and Hoefer (2007). While one can raise worries for each of these answers, these worries seem to us to be less damaging than those facing the alternatives.

\section{$4 \quad$ Extending the Account}

In the previous section, we examined several ways of developing a Best System Account of laws that does not require perfectly natural properties. We suggested that one promising route is to adopt Language-Salience and Metrics-Rigidity.

But natural properties play an important role in many Lewisian analyses duplication, intrinsicality, supervenience, materialism, among others - in addition to Lewis's account of lawhood. Can these other analyses be modified so that they too do not require perfectly natural properties?

We suggest the following strategy: specify a set of properties that can effectively play the role of the perfectly natural properties in Lewisian analyses. Call the properties in this set the surrogate properties. Wherever a Lewisian analysis invokes perfectly natural properties, substitute these surrogates. For example, instead of taking individuals to be duplicates iff there is a bijection between their parts that preserves perfectly natural properties and relations, take them to be duplicates iff there is a bijection between their parts that preserves surrogate properties and relations.

The challenge is to specify the set of surrogates in some reductionistfriendly way. Our method begins with the account of laws presented above, which adopts Language-Salience and Metrics-Rigidity. On this account, the laws are given by the set of true sentences that, given our present metrics, best balances simplicity, informativeness, and salience. Our proposal is this: the surrogate properties are all and only the properties denoted by the predicates appearing in the Best System. ${ }^{38,39,40}$

\footnotetext{
${ }^{38}$ Lewis (1983) proposes that natural properties be used as "reference magnets," to help determine the referents of predicates and names. One might worry that replacing natural properties with surrogates in an account of reference raises circularity problems. The reference magnets are the properties referred to by the predicates in the actual Best System; but we need reference magnets to determine what the referents of the predicates in the actual Best System are!

The reason this is not a problem is that the languages in which candidate systems are expressed are interpreted formal languages, and the referents of their terms are stipulated from the outset (see footnote (5)). So we don't need reference magnets to secure the referents of the predicates in the actual Best System. (What Lewis is providing is a way to determine the referents
} 


\subsection{Three Worries}

This proposal faces several challenges. Here we discuss three, in ascending order of importance.

One worry concerns the possibility of the world being very chaotic - so chaotic that there end up being few or no laws. If there are no laws, then there are no surrogate natural properties. If there are no surrogate natural properties, then none of Lewis's analyses are tenable. ${ }^{41}$ So if there are no laws, then our proposal doesn't resurrect Lewis's analyses; it renders these analyses hopeless.

We don't take this worry to be compelling. For while it's true that our world might have had few or no laws, it's not true that our world does have few or no laws. Our proposal takes the predicates that appear in our actual laws to designate surrogate properties. As long as the actual world has laws, the possibility of worlds that do not is not a threat to the account.

A second worry is that the predicates that appear in the actual Best System may denote properties that do not fit the roles Lewis envisioned. For instance, Lewis's analyses of duplication and intrinsicality entail that all the perfectly natural properties are intrinsic. Substituting surrogate properties for the perfectly natural

of terms in natural languages. Here we do need to appeal to something like reference magnets, since the referents of terms in natural languages aren't fixed by construction.) (Thanks here to Elizabeth Barnes.)

${ }^{39}$ One of the worries facing Lewis's account of laws is that several candidate systems might be tied for best, and so there might not be a unique best system. This worry might seem particularly pressing for the proposal we put forth, since the best system determines both the laws and the surrogate properties. One response to this worry is to maintain that, in the case of ties, the intersection of the properties referred to in the best systems provide the surrogates. A second response is to take the union of these properties to be the surrogates. A third response is to note that the mere possibility of ties is not a problem for this proposal, since the actual best system is used to select the surrogate properties. And, as Lewis says, "we haven't the slightest reason to think the case [of ties] really arises." (Lewis [1994] 1999, 233)

${ }^{40}$ And for quantitative properties (like three grams mass), one might want to say that for if any quantitative property appears in the Best System, then all quantitative properties belonging to the same family (e.g., mass), count as surrogates.

${ }^{41}$ Take, for instance, Lewis's analysis of duplication: two things are duplicates iff there's a bijection between their parts that preserves the perfectly natural properties and relations. If there are no surrogate natural properties, then all objects are alike with respect to their surrogates - they all have none. Together with Lewis's analysis of duplication, this entails that any mereologically isomorphic objects are duplicates. But that's clearly the wrong result. Moreover, this result will filter into any account that makes use of the notion of duplication. Consider Lewis's analysis of intrinsicality: a property is intrinsic iff it never divides duplicates. If all mereologically isomorphic objects are duplicates, then nearly every property is one that divides duplicates, and so nearly every property is extrinsic. But that, too, is the wrong result. 
ones, this entails that all the surrogate properties are intrinsic. Now consider the predicate "being five feet from an electron." If the system that best balances simplicity, informativeness, and salience employs this predicate, then this predicate denotes a surrogate property, and thus an intrinsic property. Intuitively, though, being five feet from an electron is not intrin sic.

There are two ways to resist this worry. First, it seems plausible that predicates of this sort will not appear in the actual Best System. Given LanguageSalience and Metrics-Rigidity, the best system is the one that does best at balancing our current, actual metrics of simplicity, informativeness, and salience. All else being equal, a system expressed in terms of predicates such as "being five feet from an electron," "being five feet from a proton," etc., is less simple than one that replaces these with a single relation, "being five feet from." Likewise, "being five feet from an electron" is less salient than "being five feet from;" all else being equal, a system employing the former predicate scores lower on salience than one employing the latter. Given the metrics we use to evaluate candidate systems, it seems unlikely that the system that comes out best will employ predicates that cause trouble in this way.

Second, one might say that if the Best System does include monadic predicates of this sort, then we should bite the bullet. After all, our intuitions regarding intrinsicality are not sacrosanct. For example, we typically think of charge as an intrinsic property of particles. But if the system that best balances simplicity, informativeness, and salience takes the form of certain kinds of gauge theories, then charge turns out to be extrinsic instead. ${ }^{42}$ Insofar as the actual Best System yields these kinds of unexpected results, we should take them to be discoveries, not counterexamples.

The third worry for this proposal concerns the intuitive possibility of properties that play the "naturalness" role but are not instantiated at the actual world. Call such properties foreign properties.

The proposal we've put forth has the naturalness role played by surrogate properties, and, necessarily, all surrogate properties are actual. As a result, this proposal rules out the possibility of foreign properties, and one might worry that this leads to counterintuitive results. For instance, this proposal entails that worlds alike with respect to their instantiation pattern of surrogates are duplicates. But if foreign properties are possible, then it seems that worlds alike with respect to their surrogates need not be duplicates - for one may instantiate a foreign property that the other does not.

\footnotetext{
${ }^{42}$ See Maudlin (2007, 78-103) and Arntzenius (2012, 183-193).
} 
Perhaps the best way to respond to this worry is to bite the bullet and deny the possibility of foreign properties. ${ }^{43}$ There are a few reasons one might offer for thinking that this is not much of a cost.

First, there are independent reasons why one may want to reject the possibility of foreign properties. For instance, those who want to defend a combinatorialist theory of possibility (where possible worlds are "built up" out of actual world constituents) will reject foreign properties (e.g., see Armstrong (1989)). Moreover, philosophers inclined towards the proposal we've sketched seem particularly likely to be suspicious of foreign properties. For those who dislike naturalness tend to dislike metaphysical weirdness in general, and a possible world where foreign properties are instantiated seems to fall into this category.

A second reason is that there are many possibilities concerning non-actual properties that this proposal does capture - and one might think that the demand that it also capture the possibility of foreign properties is too strong. For instance, this proposal does not rule out the possibility of alien properties (properties not instantiated at the actual world), for worlds with different instantiation patterns of surrogates may instantiate non-actual properties. Consider the property being a golden mountain one mile high. This property may very well be instantiated at some possible world, though it is alien to ours. Nor does this proposal rule out the possibility that the best system could have been different. There are possible worlds that differ from ours with respect to their instantiation patterns of surrogates, and many of these have best systems that differ from ours as well. This proposal does not even rule out the possibility that the predicates figuring in the best system could have been different. For, on this proposal, the best system of a world is the one that best balances informativeness, simplicity, and salience, evaluated using our actual metrics. This is compatible with there being a world

\footnotetext{
${ }^{43}$ If one believes that modal facts are conventional or constructed in some sense, then there may be a way to accommodate the possibility of foreign properties. Begin with a linguistic ersatzist theory, such as the one offered by Joseph Melia (2001) and (2003, 165-172). Very roughly, linguistic ersatzism is the view that possible worlds are maximal consistent sets of sentences. Let the language used to formulate the sentences have a predicate for every actual property and a name for every actual individual. Melia suggests that this language be enriched with pseudo-predicates expressions that function just like predicates but do not refer to any actual property - which can be used to represent non-actual properties. One might build on Melia's proposal by introducing a second-order pseudo-predicate, "is a foreign property," and stipulating that this pseudo-predicate satisfies certain modal constraints: (a) it applies to no actual properties, (b) it applies to some proper subset of pseudo-predicates, and (c) it applies to the same pseudo-predicates at every world. One could then introduce a disjunctive second order predicate, "is foreign or appears in the actual best system," and use this predicate to play the role of naturalness predicate in Lewis's accounts. (Similarly, one might accommodate the possibility of foreign properties by adopting a form of modal fictionalism (see Rosen (1990).)
} 
where the instantiation pattern of surrogates is such that the system that best balances informativeness, simplicity, and salience is one whose predicates do not express surrogate properties. (Suppose, for instance, that the actual best system includes the predicate expressing the property mass-density. A world with a very different pattern of mass-density properties might be more saliently described using a predicate expressing mass instead, or even a predicate expressing some alien property.) Given this, one might claim that this proposal captures all the intuitions concerning non-actual properties that one can reasonably expect.

A third reason is that there are moves one can make that mitigate the counterintuitiveness of this result. For instance, following Armstrong $(1989,73)$, one might claim that although foreign properties are not metaphysically possible, they are doxastically possible. By distinguishing metaphysical from doxastic possibility, one can attempt to capture the intuition that there could have been foreign properties by claiming that the relevant notion of "could" is epistemic. (Of course, making this move incurs the obligation to provide an account of epistemic possibility; see Chalmers (2011) for one way of doing this.)

We have sketched a proposal for how to employ the kind of naturalproperty-free account of laws suggested by Loewer and Hoefer in the more ambitious project of doing without natural properties entirely. And as we've seen, several worries can be raised for this proposal. But we believe that there are viable ways in which a proponent of this proposal could respond.

\section{$5 \quad$ Another Approach}

In the previous section, we suggested one way to modify Lewis's accounts of similarity, duplication, etc., to avoid commitment to perfectly natural properties. We began by presenting a Best System account of lawhood that did not require perfect naturalness. We then took the properties expressed by the predicates appearing in the Best System to be the "surrogate properties," and set those properties to play the role of the natural properties in Lewis's other accounts.

But there are other paths to selecting a set of surrogates. In this section, we briefly explore an alternative strategy. Instead of beginning with a Lewisian account of lawhood, this strategy begins with a Lewisian account of linguistic representation. We discuss a view presented by Williams (this volume), which modifies Lewis's account of linguistic representation to avoid appealing to primitive naturalness, and then sketch how one might employ this theory to select a set of surrogates to play the role of naturalness in other Lewisian analyses. 
Let's begin with a rough sketch of Lewis's "Best Theory" account of linguistic representation. Consider the various ways a theory may assign semantic values to expressions. Some semantic theories line up with a community's linguistic practices better than others - these theories score highly on fit. Some semantic theories assign referents that are more eligible than others - these theories score highly on eligibility. Call the semantic theory that best balances fit and eligibility the "Best Theory."

The desiderata of fit and eligibility for semantic theories are analogous to the desiderata of informativeness and simplicity for theories of lawhood. A system of lawhood that scores highly on informativeness tells us a lot about what the world is like; a semantic theory that scores highly on fit tells us a lot about what the linguistic practices of a community are like. Similarly, a system of lawhood that scores highly on simplicity is one whose axioms are relatively short and uncomplicated; a semantic theory that scores highly on eligibility is one whose axioms - which state the semantic values assigned to lexical items - are relatively short and uncomplicated. (See Williams (2007) and (this volume) for more on why the eligibility of semantic values assigned by the theory is equivalent to the simplicity of the theory's axioms. ${ }^{44}$ )

So we now have a basic template for Best Theory accounts of linguistic representation, analogous to the template for Best System accounts of lawhood. And, as before, this template is silent on two key questions. The first is the Language Question: what language should be used to formulate candidate semantic theories? Consider two theories that perfectly fit a community's patterns of use. The eligibility desideratum allows us to break the tie: the better theory is the one that assigns more eligible semantic values. But the degree to which a theory is eligible is just the degree to which its axioms are simple. And if candidate theories may be formulated in any language whatsoever, then the eligibility desideratum is too easy to satisfy - any theory can be made highly eligible, for the theory's axioms can be stated using a language that makes them as syntactically simple as you like.

\footnotetext{
${ }^{44}$ "[W] could assign a 'degree of eligibility' to each semantic value for a lexical item featuring in the semantic theory - a measure that reflects the syntactic complexity of a clause that assigns that semantic value to an expression. For example, the degree of eligibility of the property being human gives a measure of how much syntactic complexity is added to semantic theory by a clause assigning that property to the predicate 'is human'. The overall eligibility of a theory is thus just another way of measuring the syntactic complexity of that theory when spelled out in primitive terms. Measuring simplicity of a theory by its syntactic complexity when spelled out in primitive terms, or by overall eligibility of the semantic values assigned, are thus one and the same thing." (Williams 2007, 375-376)
} 
The second is the Metrics Question: which metrics should we use to evaluate candidate semantic theories? Just as there are a number of ways to assess the simplicity and informativeness of a system, there are a number of ways to assess the fit and eligibility of a theory. And which theory comes out "best" will vary depending on the metrics we employ. ${ }^{45}$

One can answer these two questions in different ways. Lewis, for instance, offers the Metrics-Objectivity answer to the Metrics Question, according to which there are objectively correct evaluative metrics of fit, eligibility, and balance; and the Language-Objectivity answer to the Language Question, according to which the language in which to formulate candidate theories is the one whose predicates express perfectly natural properties. ${ }^{46}$ In contrast, Williams (this volume) answers the Language Question in a way that avoids commitment to perfect naturalness. He offers what he calls a "parochial" version of the Best Theory account, which gives the Language-Rigidity answer to the Language Question: the language in which candidate semantic theories are formulated is a "refined, supplemented and improved" version of English, or "some interesting principled core (scientific English, or the final vocabulary of idealized science, perhaps)." (Williams follows Lewis in adopting Metrics-Objectivity.)

However one answers these two questions, the resulting semantic theory can be used to select a set of surrogate properties: formulate the semantic theory in the appropriate language, and let the surrogate properties be those expressed by the predicates appearing in its axioms. ${ }^{47}$ So we now have two strategies for selecting a set of surrogate properties - a laws-based strategy and a semantics-based strategy. Which one should we pursue?

We do not think that one has to choose a single strategy for selecting surrogate properties. Once we eliminate primitive naturalness, it is no longer important that just one thing play all the roles Lewis had naturalness play. ${ }^{48}$

\footnotetext{
${ }^{45}$ As has been frequently noted, characterizing simplicity purely in terms of syntactic complexity, together with the assumption that the predicates that figure in the semantic theory also figure in fundamental physical theories, yields implausible results. See Schaffer (2004), Hawthorne (2006, 205-206), and Williams (this volume).

${ }^{46}$ See Lewis (1975) for remarks on fit, Lewis ([1984] 1999, 65-66) for remarks on eligibility, and Lewis ([1983] 1999, 47-48 and 51-54) for remarks on both fit and eligibility. See also Williams (this volume).

${ }^{47}$ How well these surrogates fit into Lewis's accounts will depend on how one answers the Language and Metrics Questions. While we are not here tackling the issue of which answers are most promising for the best theory account of linguistic representation, much of our discussion in section (3) applies here as well.

${ }^{48}$ For an in-depth discussion of the (in)compatibility of the many roles Lewis has naturalness play, see Dorr and Hawthorne (2014).
} 
Plausibly, the surrogates selected using the laws-based strategy are better suited to some of the naturalness roles, while the surrogates selected using the semanticsbased strategy are better suited to others. For instance, one might think that the surrogates selected using the laws-based strategy are well-suited to play the role of naturalness in analyses of duplication, intrinsicality, and related notions; while the surrogates selected using the semantics-based strategy are well-suited to play the role of naturalness as reference magnets. Alternatively, one might combine the two strategies. One way is to take the set of surrogate properties to be the union of those selected using the laws-based strategy and the semantics-based strategy. Another way is to take candidate theories to be pairs of lawhood and semantic theories - a "laws-and-semantics" package - and then take the surrogate properties to be those that appear in the best laws-and-semantics pair. Which of these options is best is a question for further research.

\section{Conclusion}

The prospect of avoiding commitment to perfectly natural properties while holding on to a Lewisian analysis of lawhood is appealing to many philosophers. In this paper, we explored different ways one might pursue this project, and argued that a Best System account that adopts Language-Salience and Metrics-Rigidity, along the lines suggested by Loewer (2007) and Hoefer (2007), has the most hope of success. On this version of the Best System account of lawhood, the best system is formulated in the language of the candidate system that best balances simplicity, informativeness, and salience - where metrics of simplicity, informativeness, salience, and balance are our current, actual metrics. We then explored the possibility of modifying other Lewisian analyses, so that they too avoid commitment to a primitive distinction among properties. We suggested that the version of the Best System account developed in section (3) can be used to specify a set of "surrogate properties" - properties that can be substituted for perfectly natural properties in other Lewisian analyses.

Of course, there are several worries one might raise for this kind of proposal. But none of them seem insurmountable, and we've suggested some ways in which a proponent can respond. Given this, we take this proposal to be an interesting and viable way to maintain that there is no work for a theory of universals. ${ }^{49}$

\footnotetext{
${ }^{49}$ Many thanks to participants of the UC-Davis DEX Conference, the Midsummer Philosophy Workshop in Edinburgh, the Fundamentality Workshop in Birmingham, and audience members at New York University for helpful discussion. Thanks in particular to Elizabeth Barnes, Sara
} 


\section{References}

Armstrong, D.M. 1989. A Combinatorial Theory of Possibility. Cambridge University Press.

Arntzenius, Frank. 2012. Space, Time and Stuff. Oxford University Press.

Callender, Craig and Jonathan Cohen. 2009. "A Better Best System Account of Lawhood." Philosophical Studies 145 (1): 1-34.

Carroll, John. 1990. “The Humean Tradition.” The Philosophical Review 99 (2): 185-219.

Carroll, John. 1994. Laws of Nature. Cambridge University Press.

Chalmers, David. 2011. “The Nature of Epistemic Space.” In Andy Egan and Brian Weatherson, eds., Epistemic Modality. Oxford University Press.

Dorr, Cian and John Hawthorne. 2014. "Naturalness." Oxford Studies in Metaphysics, Vol. 8. Oxford University Press.

Halpin, John F. 2003. "Scientific Law: A Perspectival Account." Erkenntnis 58 (2): 137-168.

Hawthorne, John. 2006. "Epistemicism and Semantic Plasticity." Oxford Studies in Metaphysics, Vol. 2. Reprinted in Hawthorne, Metaphysical Essays, pg. 185210.

Hoefer, Carl. 2007. "The Third Way on Objective Probability: A Skeptic's Guide to Objective Chance." Mind 116: 549-596.

Kratzer, Angelika. 1986. “Conditionals.” Chicago Linguistics Society 22 (2): 1-15.

Lange, Marc. 2009. Laws and Lawmakers: Science, Metaphysics, and the Laws of Nature. Oxford University Press.

Lewis, David. 1979. “Counterfactual dependence and time’s arrow.” Nô̂s 13 (4): 455-476.

Bernstein, Phil Bricker, Tim Button, Louis deRosset, Sinan Dogramaci, Janice Dowell, Jesse Fitts, Shieva Kleinschmidt, Karen Lewis, Daniel Nolan, Zee Perry, Adam Sennet, Catherine Sutton, Lee Walters, and Alastair Wilson. Special thanks to John Roberts for extraordinarily extensive and helpful comments. Finally, this paper owes an especially large debt to Barry Loewer. As will be clear to anyone who has talked with Loewer about these issues, this paper is largely an exploration of his ideas. 
Lewis, David. 1980. “A Subjectivist's Guide to Objective Chance.” In Richard Jeffery, ed., Studies in Inductive Logic and Probability, Volume II. University of California Press. Reprinted with postscripts in Philosophical Papers, Volume II, pg. 83-132.

Lewis, David. 1983. "New Work for a Theory of Universals." Australasian Journal of Philosophy 61: 343-377. Reprinted in Papers in Metaphysics and Epistemology, pg. 8-55.

Lewis, David. 1984. "Putnam's Paradox.” Australasian Journal of Philosophy 62: 221-236. Reprinted in Papers in Metaphysics and Epistemology, pg. 56-77.

Lewis, David. 1986. Philosophical Papers, Volume II. Oxford University Press.

Lewis, David. 1994. “Humean Supervenience Debugged.” Mind 103: 473-490. Reprinted with postscripts in Papers in Metaphysics and Epistemology, pg. 224-247.

Lewis, David. 1999. Papers in Metaphysics and Epistemology. Cambridge University Press.

Lewis. David. 2001. "Redefining 'Intrinsic'." Philosophy and Phenomenological Research 63: 381-398.

Loewer, Barry. 2001. "Determinism and Chance." Studies in the History of Modern Physics 32: 609-620.

Loewer, Barry. 2007. "Laws and Natural Properties.” Philosophical Topics 35 (1/2): 313-328.

MacFarlane, John. 2005. "Making sense of relative truth." Proceedings of the Aristotelian Society 105 (3): 321-339.

MacFarlane, John. 2009. "Epistemic modals are assessment-sensitive.” In Andy Egan \& Brian Weatherson, eds., Epistemic Modality. Oxford University Press.

MacFarlane, John. $2013 a$ (draft). Assessment Sensitivity: Relative Truth and Its Applications.

MacFarlane, John. 2013 b (draft). "Relativism and Knowledge Attributions." Maudlin, Tim. 2007. The Metaphysics Within Physics. Oxford University Press. Meacham, Christopher J. G. 2014. "Chance and the Conflicts Problem.” In Alastair Wilson, ed., Chance and Temporal Asymmetry. Oxford University Press. 
Melia, Joseph. 2001. "Reducing Possibilities to Language.” Analysis 61 (1): 19-29.

Melia. Joseph. 2003. Modality. McGill-Queen’s University Press.

Roberts, John. 1999. "Laws of Nature' as an Indexical Term: A Reinterpretation of Lewis's Best-System Analysis.” Philosophy of Science 66 (3): S502-S511.

Roberts, John. 2009. The Law-Governed Universe. Oxford University Press.

Rosen, Gideon. 1990. “Modal Fictionalism.” Mind 99 (395): 327-254.

Schaffer, Jonathan. 2004. "Two Conceptions of Sparse Properties.” Pacific Philosophical Quarterly 85 (1): 92-102.

Taylor, Barry. 1993. “On Natural Properties in Metaphysics.” Mind 102 (405): 81100.

Williams, J. R. G. 2007. "Eligibility and Inscrutability.” Philosophical Review 108 (3): 361-399.

Williams, J. R. G. (this volume). "Lewis on Reference and Eligibility.” In Barry Loewer and Jonathan Schaffer, eds., Companion to David Lewis. Blackwell.

Winsberg, Eric. 2008. "Laws and Chances in Statistical Mechanics." Studies in History and Philosophy of Modern Physics 39: 872-888. 